CORRECTION

\title{
Correction to: Unexpected myriad of co-occurring viral strains and species in one of the most abundant and microdiverse viruses on Earth
}

Francisco Martinez-Hernandez (D), Awa Diop (D), Inmaculada Garcia-Heredia, Louis-Marie Bobay (D) and Manuel Martinez-Garcia (iD)

(c) The Author(s), under exclusive licence to International Society for Microbial Ecology 2021

The ISME Journal (2022) 16:1199; https://doi.org/10.1038/s41396-021-01182-8

Correction to: The ISME Journal https://doi.org/10.1038/s41396021-01150-2

The Acknowledgements section was given incomplete in this article and should have read: This work has been supported by the Spanish Ministry of Science and Innovation (RTI2018-094248-B100), Gordon and Betty Moore Foundation (grant 5334) and Generalitat Valenciana (ACIF/2015/332 and APOSTD/2020/237). This work was also supported by the National Science Foundation under Grant No. DEB-1831730 awarded to L.M.B. and by the
National Institute of General Medical Sciences of the National Institutes of Health under Award Number R01GM132137 awarded to L.M.B.

We thank Dr. Josep Gasol for giving us access to collecting samples from REMEl Expedition and Dr. Mario Martinez-Lopez for sharing a collection of Pelagibacter genomes.

The original article has been corrected. 